\title{
Non-Liouvillian Solutions for Second Order Linear ODEs
}

\author{
L. Chan \\ Department of Pure Mathematics \\ University of Waterloo \\ Waterloo, Ontario, Canada, N2L 3G1 \\ kclchan@pythagoras.math.uwaterloo.ca
}

\author{
E.S. Cheb-Terrab \\ CECM, Department of Mathematics \\ Simon Fraser University \\ Vancouver, British Columbia, Canada, V5A 1S6 \\ Maplesoft, Waterloo Maple Inc. \\ Waterloo, Ontario, Canada, N2V 1K8 \\ ecterrab@cecm.sfu.ca
}

\begin{abstract}
There exist sound literature and algorithms for computing Liouvillian solutions for the important problem of linear ODEs with rational coefficients. Taking as sample the 363 second order equations of that type found in Kamke's book, for instance, $51 \%$ of them admit Liouvillian solutions and so are solvable using Kovacic's algorithm. On the other hand, special function solutions not admitting Liouvillian form appear frequently in mathematical physics, but there are not so general algorithms for computing them. In this paper we present an algorithm for computing special function solutions which can be expressed using the ${ }_{2} \mathrm{~F}_{1},{ }_{1} \mathrm{~F}_{1}$ or ${ }_{0} \mathrm{~F}_{1}$ hypergeometric functions. The algorithm is easy to implement in the framework of a computer algebra system and systematically solves $91 \%$ of the 363 Kamke's linear ODE examples mentioned.
\end{abstract}

\section{Categories and Subject Descriptors}

I.1 [Symbolic and algebraic manipulation]: Algorithms.

\section{General Terms}

Algorithms, design, theory.

\section{Keywords}

Linear ordinary differential equations, Non-Liouvillian solutions, hypergeometric solutions.

\section{Introduction}

Given a second order linear ODE

$$
y^{\prime \prime}+A(x) y^{\prime}+B(x) y=0
$$

where the quantity ${ }^{1} A^{\prime} / 2+A^{2} / 4-B$ is a rational function of $x$, the problem under consideration is that of systematically

\footnotetext{
${ }^{1}$ This quantity is an invariant under transformations of the dependent variable - see (12).
}

Permission to make digital or hard copies of all or part of this work for personal or classroom use is granted without fee provided that copies are not made or distributed for profit or commercial advantage and that copies bear this notice and the full citation on the first page. To copy otherwise, to republish, to post on servers or to redistribute to lists, requires prior specific permission and/or a fee.

ISSAC'04, July 4-7, 2004, Santander, Spain.

Copyright 2004 ACM 1-58113-827-X/04/0007 ...\$5.00. computing solutions for this ODE even when the solutions admit no Liouvillian form ${ }^{2}$.

The first thing to note is that non-Liouvillian solutions which are representable symbolically not as unknown infinite sums can be represented using special functions, e.g. Bessel, Hermite or Legendre functions [1]. In turn, these and most of the special functions frequently appearing in mathematical physics happen to be particular cases of the ${ }_{p} \mathrm{~F}_{q}$ hypergeometric function for $p$ equal to 0,1 or 2 and $q$ equal to 0 or 1 (see 2 ). For example, the Bessel functions can be expressed in terms of ${ }_{0} \mathrm{~F}_{1}$, all cylindrical functions as well as the Hermite, Laguerre, Whittaker and error family of functions can be expressed in terms of ${ }_{1} \mathrm{~F}_{1}$, and all Chebyshev, Gegenbauer, Jacobi, Legendre and some others can be expressed in terms of ${ }_{2} \mathrm{~F}_{1}$.

One natural approach is then to directly attempt the computation of hypergeometric function solutions of these ${ }_{0} \mathrm{~F}_{1}$, ${ }_{1} \mathrm{~F}_{1}$ and ${ }_{2} \mathrm{~F}_{1}$ types, since in this way we cover at once solutions involving all the related special functions. Such an approach was developed during the year 2001 (see [3]), it became the main algorithm of the Maple computer algebra system for this type of problem since then and it is the subject of this paper. The algorithm consists of an equivalence approach to the ${ }_{p} \mathrm{~F}_{q}$ differential equations, is formulated in sec.11 2 and 3, and computes solutions of the form

$$
y=P(x){ }_{p} \mathrm{~F}_{q}\left(. . ; . . ; \frac{\alpha x^{k}+\beta}{\gamma x^{k}+\delta}\right)
$$

where $P(x)$ is an arbitrary function and $\{\alpha, \beta, \gamma, \delta, k\}$ are constants.

It is important to note that the idea of seeking hypergeometric function solutions for (1) or using an equivalence approach for that is not new. In '89 Kamran and Olver [4] showed how to use an equivalence approach to compute Bessel function solutions to eigenvalue problems. Hypergeometric solutions were also discussed by Petkovsek and Salvy [5] in '93. Some of the more recent developments were presented as computer algebra algorithms too. For instance, a classic invariant theory approach was presented during 2000 by von Bülow in [6]; in 2001 Willis [7] presented a semi-heuristic algorithm for computing special functions

\footnotetext{
${ }^{2}$ Functions that can be expressed in terms of exponentials, integrals, and algebraic functions, are called Liouvillian functions. The typical example is $\exp \left(\int R(x), d x\right)$ where $R(x)$ is rational or an algebraic function representing the roots of a polynomial.
} 
solutions. In 2002 Bronstein and Lafaille 8] presented an approach for resolving an equivalence under rational transformations, between two linear equations in normal form, whenever one of them has an irregular singularity ${ }^{3}$.

There is natural intersection between what these algorithms can solve but none can claim to extensively cover the portions of the problem covered by all the others. If compared with the algorithm presented in this paper - we called it hyper3 - these other algorithms, both those developed before and after hyper3:

- Do not resolve in a systematic manner all of the ${ }_{2} \mathrm{~F}_{1}$, ${ }_{1} \mathrm{~F}_{1}$ and ${ }_{0} \mathrm{~F}_{1}$ equivalences;

- Do not handle the problem of an invariant involving fractional or abstract powers;

- Do not explore automorphisms to avoid uncomputed integrals in the solution.

Also, hyper3 does not require solving systems of algebraic equations nor computing Groebner basis nor running differential elimination processes nor eliminating parameters by composing resultants (all of them expensive computational processes), thus resulting in a fast and smooth algorithm with little computational cost. These facts, combined with the range of problems it solves, for instance taking Kamke's book 12 as a testing arena, are at the base of the role hyper3 has today in the Maple differential equation libraries.

\section{COMPUTING ${ }_{2} \mathbf{F}_{1},{ }_{1} \mathbf{F}_{1}$ AND ${ }_{0} \mathbf{F}_{1}$ HYPER- GEOMETRIC SOLUTIONS}

To compute ${ }_{p} \mathrm{~F}_{q}$ solutions to (1), the idea is to formulate an equivalence approach to the ${ }_{p} \mathrm{~F}_{q}$ underlying hypergeometric differential equations; that is, to determine whether a given linear ODE can be obtained from one of the ${ }_{2} \mathrm{~F}_{1}$, ${ }_{1} \mathrm{~F}_{1}$ or ${ }_{0} \mathrm{~F}_{1}$ ODEs, respectively given by

$$
\begin{aligned}
\left(x^{2}-x\right) y^{\prime \prime}+((a+b+1) x-c) y^{\prime}+b a y & =0 \\
x y^{\prime \prime}+(c-x) y^{\prime}-a y & =0 \\
x y^{\prime \prime}+c y^{\prime}-y & =0
\end{aligned}
$$

where $\{a, b, c\}$ are arbitrary constants, by means of a transformation of a certain type. If so, the solution to the given linear ODE is obtained by applying the same transformation to the solution of the corresponding ${ }_{p} \mathrm{~F}_{q}$ ODE above.

This approach of course also requires determining the values of the hypergeometric parameters $\{a, b, c\}$ for which the equivalence exists, and it is clear that its chances of success depend crucially on how general is the class of transformations being considered. For instance, one can verify that for linear transformations ${ }^{4}$

$$
x \rightarrow F(x), \quad y \rightarrow P(x) y
$$

with arbitrary $F(x), P(x)$, the problem is too general in that to solve it requires solving first the given ODE, so that the approach is of no practical use [6].

\footnotetext{
${ }^{3}$ That also leads to ${ }_{1} \mathrm{~F}_{1}$ solutions of the form (2), including its particular ${ }_{0} \mathrm{~F}_{1}$ case, whenever the point of application of ${ }_{1} \mathrm{~F}_{1}$ is rational in the independent variable.

${ }^{4}$ The problem of equivalence under transformations $\{x \rightarrow$ $F(x), \quad y \rightarrow P(x) y+Q(x)\}$ for linear ODEs can always be mapped into one with $Q(x)=0$, see [9].
}

The transformations considered in this work are

$$
x \rightarrow \frac{\alpha x^{k}+\beta}{\gamma x^{k}+\delta}, \quad y \rightarrow P(x) y
$$

with $P(x)$ arbitrary and $\{\alpha, \beta, \gamma, \delta, k\}$ constant with respect to $x$. These transformations, which do not conform a class in the strict sense ${ }^{5}$, can be obtained by sequentially composing three different transformations each of which does constitute a class. The sequence starts with linear fractional - also called Möbius - transformations

$$
M:=x \rightarrow \frac{\alpha x+\beta}{\gamma x+\delta},
$$

is followed by power transformations

$$
x \rightarrow x^{k}
$$

and ends with linear homogeneous transformations of the dependent variable

$$
y \rightarrow P y
$$

So, we are talking of an algorithm that systematically computes, when they exist, solutions of the form

$$
y=P(x){ }_{p} \mathrm{~F}_{q}\left(. . ; . ; \frac{\alpha x^{k}+\beta}{\gamma x^{k}+\delta}\right)
$$

where ${ }_{p} \mathrm{~F}_{q}$ is any of ${ }_{2} \mathrm{~F}_{1},{ }_{1} \mathrm{~F}_{1}$ or ${ }_{0} \mathrm{~F}_{1}$.

\subsection{Transformations $y \rightarrow P(x) y$ of the depen- dent variable}

The first thing to note is that transformations of the form (8) can easily be factored out of the problem: if two equations of the form (1), with coefficients $\{A(x), B(x)\}$ and $\{C(x), D(x)\}$ respectively, can be obtained from each other by means of (8), the transformation relating them is computable from these coefficients. For that purpose, we rewrite both equations in normal form, for instance for (1) use

$$
y=u e^{-\int A / 2 d x}
$$

to obtain

$$
u^{\prime \prime}=\left(\frac{A^{\prime}}{2}+\frac{A^{2}}{4}-B\right) u
$$

and the transformation relating the two hypothetical ODEs exists when the two normalized equations are equal; the transformation relating them being $y=u e^{\int(C-A) / 2 d x}$. In what follows we will refer to

$$
I(x)=\frac{A^{\prime}}{2}+\frac{A^{2}}{4}-B,
$$

the coefficient of $u$ in (11), as the invariant 10, regardless of the fact that this object is only an absolute invariant under (8) and not under (6) or (7).

\footnotetext{
${ }^{5} \mathrm{By}$ class of transformations we mean a set of transformations closed under composition.
} 


\subsection{Transformations $x \rightarrow F(x)$ of the indepen- dent variable}

By changing $x \rightarrow F(x)$ in (1), the invariant $I_{1}$ of the changed ODE can be expressed in terms of the invariant $I_{0}$ of (1) by

$$
I_{1}(x)=F^{\prime 2} I_{0}(F(x))+S\left(F^{\prime}\right)
$$

where $S(x)$ is the Schwarzian 11

$$
S\left(F^{\prime}\right)=\frac{3 F^{\prime \prime 2}}{4 F^{\prime 2}}-\frac{F^{\prime \prime \prime}}{2 F^{\prime}}
$$

The form of $S\left(F^{\prime}\right)$ is particularly simple when $F(x)$ is a power transformation (see (23)) and also when $F(x)$ is a Möbius transformation (6), in which case $S\left(F^{\prime}\right)=0$. These are key facts permitting a simple formulation and resolution of the equivalence.

\section{MÖBIUS TRANSFORMATIONS AND A CLASSIFICATION OF SINGULARITIES}

The first ODE in (3) has 3 regular singularities, at 0,1 and $\infty$. The second ODE in (3), also known as the confluent hypergeometric equation, has a regular singularity at 0 and an irregular one at $\infty$. The third ODE in (3) also has one regular and one irregular singularity at 0 and $\infty$, but we considered the case separately in order to obtain solutions directly expressed in terms of simpler (Bessel) functions. As we shall see, the structure of the singularities of these equations is a key for resolving related equivalences and Möbius transformations preserve that structure. These transformations only move the location of the poles. For example, the ${ }_{0} \mathrm{~F}_{1}$ hypergeometric equation

$$
x y^{\prime \prime}+c y^{\prime}-y=0
$$

has one regular singularity at the origin and one irregular at infinity. The transformed ODE, obtained from (15) by means of (6)

$$
\begin{array}{r}
y^{\prime \prime}+\frac{(\alpha(\delta c+2 \gamma x)+\gamma(2-c) \beta)}{(\alpha x+\beta)(\gamma x+\delta)} y^{\prime} \\
-\frac{(\alpha \delta-\gamma \beta)^{2}}{(\gamma x+\delta)^{3}(\alpha x+\beta)} y=0
\end{array}
$$

also has one regular and one irregular singularity, respectively located at $-\beta / \alpha$ and $-\delta / \gamma$. In the case of the ${ }_{2} \mathrm{~F}_{1}$ equation (see (3)), under (6) the three regular singularities move from $\{0,1, \infty\}$ to $\{-\delta / \gamma,-\beta / \alpha,(\delta-\beta) /(\alpha-\gamma)\}$. So, from the structure of the singularities of an ODE, not only one can tell with respect to which of the three differential equations (3) could the equivalence under (6) be resolved, but also one can extract information regarding the values of the parameters $\{\alpha, \beta, \gamma, \delta\}$ entering the transformation.

Reversing the line of reasoning, through Mobius transformations one can formulate a classification of singularities of the linear ODEs "equivalent" to the ${ }_{p} \mathrm{~F}_{q}$ equations (3), based on how the invariant of each of these equations is transformed. Concretely, after transforming the ${ }_{2} \mathrm{~F}_{1}$ equation, the invariant of the resulting equation has the form

$$
I_{2} \mathrm{~F}_{1}=\frac{\omega_{2} x^{2}+2 \omega_{1} x+\omega_{0}}{\left(\sigma_{1} x+\sigma_{2}\right)^{2}\left(\sigma_{3} x+\sigma_{4}\right)^{2}\left(\sigma_{5} x+\sigma_{6}\right)^{2}}
$$

where all $\left\{\omega_{i}, \sigma_{j}\right\}$ can be expressed in terms of $\{a, b, c\}$ and $\{\alpha, \beta, \delta, \gamma\}$ respectively entering the ${ }_{2} \mathrm{~F}_{1}$ equation (3) and the transformation (6). The invariant of the transformed ${ }_{1} \mathrm{~F}_{1}$ equation has the form

$$
I_{1} \mathrm{~F}_{1}=\frac{\omega_{2} x^{2}+2 \omega_{1} x+\omega_{0}}{\left(\sigma_{3} x+\sigma_{4}\right)^{2}\left(\sigma_{5} x+\sigma_{6}\right)^{4}}
$$

and that of the transformed ${ }_{0} \mathrm{~F}_{1}$ equation has the form

$$
I_{0} \mathrm{~F}_{1}=\frac{\omega_{1} x+\omega_{0}}{\left(\sigma_{3} x+\sigma_{4}\right)^{2}\left(\sigma_{5} x+\sigma_{6}\right)^{3}}
$$

These transformed invariants are all of the form

$$
I_{p} \mathrm{~F}_{q}=\frac{\prod_{i=1}^{m}\left(a_{i} x+b_{i}\right)}{\prod_{i=1}^{n}\left(c_{i} x+d_{i}\right)^{q_{i}}}
$$

Cancellations between factors in the numerator and denominators of (20) may also happen and, independent of that, some coefficients $\left\{a_{i}, c_{i}\right\}$ can be zero ${ }^{6}$. So the degrees with respect to $x$ of the numerators and denominators of (17), (18) and (19) can be lower than the maximum implicit by these equations; in this way the problem splits into cases.

Taking these possible cancellations into account, from the structure of the invariants (17), (18) and (19), the different cases for each of the ${ }_{2} \mathrm{~F}_{1},{ }_{1} \mathrm{~F}_{1},{ }_{0} \mathrm{~F}_{1}$ classes were determined. With this classification in hands, from the knowledge of the degrees with respect to $x$ of the numerator and denominator of the invariant (20) of a given ODE, one can tell whether or not it can be obtained from the ${ }_{2} \mathrm{~F}_{1},{ }_{1} \mathrm{~F}_{1}$ or ${ }_{0} \mathrm{~F}_{1}$ equations (3) using (6). These observations can be summarized in a classification table as follows, using the symbol

$$
\left[\leq p,\left[q_{1} *, q_{2} *, \ldots, q_{n} *\right]\right]
$$

where $p$ is the degree in $x$ of the numerator of (20) and $q_{i}$ are the powers of the factors entering the denominator of it. The symbol $\leq$, when present, refers to the value of $p$ (can be less or equal to). The symbol $*$, when present, means there can be factors canceling between numerator and denominator, so that the actual value of the related $q_{i}$ can be lower (provided $p$ is also lower by the same amount). For example,

$$
[\leq 2 *,[2 *, 2 *]]
$$

represents the following possible seven different "lists of values" (herein referred as cases) for the degrees of the numerator and denominator of the invariant

$$
\begin{aligned}
{[2 *,[2 *, 2 *]] } & =[2,[2,2]],[1,[1,2]],[0,[1,1]],[0,[0,2]] \\
{[1 *,[2 *, 2 *]] } & =[1,[2,2]],[0,[1,2]] \\
{[0,[2,2]] } &
\end{aligned}
$$

With this notation, the classification of all the possible cases equivalent to the ${ }_{2} \mathrm{~F}_{1},{ }_{1} \mathrm{~F}_{1}$ and ${ }_{0} \mathrm{~F}_{1}$ equations under Möbius transformations is as shown in Table 1.

\footnotetext{
${ }^{6}$ Provided that, in (6), $\alpha \delta-\gamma \beta \neq 0$ and also that in (1) the invariant remains finite, i.e. its denominator is not zero.
} 


\begin{tabular}{|c|l|c|}
\hline Class & Cases & Number of cases \\
\hline${ }_{2} \mathrm{~F}_{1}$ & {$[<=2 *,[2 *, 2 *, 2 *]],[<=2 *,[2 *, 2 *]]$} & 14 \\
\hline${ }_{1} \mathrm{~F}_{1}$ & {$[2 *,[2 *, 4]],[<=2,[6]],[<=2,[4]],[2 *,[2 *]],[2,[0]]$} & 13 \\
\hline${ }_{0} \mathrm{~F}_{1}$ & {$[1 *,[2 *, 3]],[<=1,[5]],[<=1,[3]],[1 *,[2 *]],[1,[0]]$} & 9 \\
\hline
\end{tabular}

Table 1. Classification of linear ODEs equivalent to ${ }_{p} F_{q}$ ODEs under Möbius

\section{TRANSFORMATIONS $x \rightarrow X^{K}$ OF THE INDEPENDENT VARIABLE}

Using the results of the previous sections it is possible to resolve the equivalence of a given linear ODE (1) and the hypergeometric equations (3) under compositions of transformations (8) of the dependent variable $y(x)$ and Möbius transformations (6) of the independent variable $x$. In this section a worth additional level of generalization is obtained by composing those two transformations with transformations $x \rightarrow x^{k}$ of the independent variable.

The first thing to note regarding power transformations is that, unlike Möbius transformations, they do not preserve the structure of singularities. The change in the invariant due to $x \rightarrow x^{k}$, however, has a simple and tractable structure. The Schwarzian (14) is given by:

$$
S\left(F^{\prime}\right)=\frac{k^{2}-1}{4 x^{2}}
$$

So, the changed invariant $I_{1}$ shown in (13) can be expressed in terms of $I_{0}$ by

$$
x^{2} I_{1}(x)+\frac{1}{4}=\left(\left(x^{k}\right)^{2} I_{0}\left(x^{k}\right)+\frac{1}{4}\right) k^{2}
$$

This naturally suggests the introduction of a "shifted" invariant $J(x)$

$$
J_{i}(x)=x^{2} I_{i}(x)+\frac{1}{4}
$$

for which the transformation rule under $x \rightarrow x^{k}$ has the simple form

$$
J_{1}(x)=k^{2} J_{0}\left(x^{k}\right)
$$

The equivalence of two linear ODEs $\mathrm{A}$ and $\mathrm{B}$ under $x \rightarrow$ $x^{k}$ can then be formulated as follows: Given $J_{1 A}(x)$ and $J_{1 B}(x)$, compute $k_{A}$ and $k_{B}$ entering (26) such that the degrees with respect to $x$ of $J_{0 A}(x)$ and $J_{0 B}(x)$ are minimized. This approach is systematic: equations $\mathrm{A}$ and $\mathrm{B}$ are related through power transformations only when $J_{0 A}=J_{0 B}$ and, if so, the mapping relating $\mathrm{A}$ and $\mathrm{B}$ is just $x \rightarrow x^{k_{A}-k_{B}}$.

The computation of $k$ minimizing the degrees of $J_{0}$ in (26) is formulated as follows. Given the set

$$
A:=\frac{p_{i}}{q_{i}}, \quad i=1 \text { to } m
$$

of (possibly rational) numbers entering as exponents in the powers of the independent variable found in $J_{1}$, compute the smallest rational number $\tilde{k}$ such that multiplying by it each element of $A$, all of them become integers. Then the value of $k$ minimizing the degrees of $J_{0}$ is $k=1 / \tilde{k}$.

\section{SUMMARY OF HYPER3 - EXAMPLES}

An itemized description of the algorithm, discussed in the previous subsections to resolve the equivalence proposed in the introduction, is as follows.

1. Rewrite the given equation (1) we want to solve in normal form

$$
y^{\prime \prime}=I(x) y
$$

where $I(x)$ is the invariant (12).

2. Compute $J_{1}(x)$, the shifted invariant (25), and use transformations $x \rightarrow x^{k}$ to reduce to the integer minimal values the exponents of powers entering $J_{0}(x)$; i.e., compute $k$ and with it compute $J_{0}(x)$ in (26).

3. From (25), compute $I_{0}(x)$ and classify its structure of singularities according to Table 1, to tell whether an equivalence under Möbius transformations is possible and to which of the ${ }_{2} \mathrm{~F}_{1},{ }_{1} \mathrm{~F}_{1}$ or ${ }_{0} \mathrm{~F}_{1}$ equations (3).

4. When the equivalence is possible, from the singularities of $I_{0}(x)$ and by comparing it with the invariant (20) of the transformed ${ }_{p} \mathrm{~F}_{q}$ equation ${ }^{7}$, compute the parameters $\{a, b, c\}$ entering the ${ }_{p} \mathrm{~F}_{q}$ equation (3) such that the equivalence exists as well as the parameters $\{\alpha, \beta, \gamma, \delta\}$ entering the Möbius transformation (6).

5. Compose the three transformations to obtain one of the form

$$
x \rightarrow \frac{\alpha x^{k}+\beta}{\gamma x^{k}+\delta}, \quad y \rightarrow P(x) y
$$

mapping the ${ }_{p} \mathrm{~F}_{q}$ equation involved into the ODE being solved.

6. Apply this transformation to the known solution of the ${ }_{p} \mathrm{~F}_{q}$ equation resulting in the desired ODE solution.

\section{An example of the ${ }_{2} F_{1}$ class}

Consider the second order linear ODE

$$
\begin{aligned}
y^{\prime \prime}= & \frac{2(\nu-\mu) x^{2}-3 x^{4}-2(\mu+\nu)-1}{x^{5}-x} y^{\prime} \\
& +\frac{\nu(\nu+2(\mu+1))}{x^{6}-x^{2}} y
\end{aligned}
$$

This equation has regular singularities at $\{0,1,-1, i,-i\}$. Following the steps outlined in the Summary, we rewrite the equation in normal form and then compute the value

\footnotetext{
${ }^{7}$ At this point, $J_{0}(x)$ and the shifted invariant of the
} ${ }_{p} \mathrm{~F}_{q}$ equation have the same degrees. 
of $k$ leading to an equation with minimal degrees for the powers entering $J_{0}(x)$ in (26). The value found is $k=2$. So, using $^{8}$

$$
t=x^{2}, \quad u=\sqrt{x} \mathrm{e}^{\left(\int \frac{2(\nu-\mu) x^{2}-3 x^{4}-2(\mu+\nu)-1}{2\left(x-x^{5}\right)} d x\right)} y,
$$

the given equation (29) can be obtained from

$$
u^{\prime \prime}=\frac{\left(\mu^{2}+2\left(\nu^{2}-\mu-2\right)\right) t^{2}+2\left(\mu^{2}-\nu^{2}\right) t+\mu(\mu+2)}{4 t^{2}(t-1)^{2}(t+1)^{2}} u,
$$

which is in normal form and has an invariant with "minimal degrees" with respect to power transformations (7).

In step 3, analyzing the invariant of (31) (coefficient of $u$ in its right-hand-side), the equation has now three regular singular points, at $\{0,1,-1\}$. Using the notation of sec.1.2 the degrees with respect to $t$ of the numerator and of each of the linear factors entering the denominator are $[2,[2,2,2]]$. The equation matches the classification Table 1 presented in sec. 1.2 and is identified as equivalent to the ${ }_{2} \mathrm{~F}_{1}$ equation under Möbius transformations (6).

So we proceed with step 4 , equating the invariant of 31 with the invariant (17) written in terms of $\{a, b, c, \alpha, \beta, \gamma, \delta\}$, from where we compute the values of the hypergeometric parameters $\{a, b, c\}$ entering the ${ }_{2} \mathrm{~F}_{1}$ equation (3), such that the equivalence under Möbius exists, as well as the Möbius transformation itself, obtaining

$$
\left\{a=\frac{\nu}{2}, b=\frac{\nu}{2}-\mu, c=-\mu\right\} \quad M:=x=\frac{2 t}{t-1}
$$

The transformation mapping the ${ }_{2} \mathrm{~F}_{1}$ equation (3) at these values of the parameters $\{a, b, c\}$ into (BI) is then obtained composing the Möbius transformation above with one of the form (8), computed as explained in sec.1.1 resulting in

$$
x=\frac{2 t}{t-1}, \quad y=\frac{t^{\mu / 2}(t-1)^{(\nu-\mu-1) / 2}}{(t+1)^{(\nu+1) / 2}} u(t)
$$

At this point, we have the transformation (32) mapping (3) into (31), and the transformation 30, mapping (31) into the equation (29) we want to solve. Composing these transformations, in step six we obtain the solution of (29)

$$
\begin{aligned}
y= & \frac{x^{\nu}}{\left(x^{2}-1\right)^{\frac{\nu}{2}}}{ }_{2} \mathrm{~F}_{1}\left(\frac{\nu}{2}, \frac{\nu}{2}-\mu ;-\mu ; \frac{2 x^{2}}{x^{2}-1}\right) C_{1} \\
+ & \frac{x^{\nu+2 \mu+2}}{\left(x^{2}-1\right)^{1+\mu+\nu / 2}} \\
& { }_{2} \mathrm{~F}_{1}\left(\frac{\nu}{2}+1,1+\mu+\frac{\nu}{2} ; 2+\mu ; \frac{2 x^{2}}{x^{2}-1}\right) C_{2}
\end{aligned}
$$

where $C_{1}$ and $C_{2}$ are arbitrary constants.

As mentioned in the introduction, an implementation of the algorithm being presented is at the core of the current Maple ability to solve this type of problem. The time consumed by this Maple implementation to compute the solution (33) performing all the steps mentioned is 0.4 seconds

\footnotetext{
${ }^{8}$ This transformation is the composition of $t \equiv x^{k}=x^{2}$ with a transformation of the form (10) so that (31) is normalized.
}

in a Pentium IV, 2 GigaHertz computer. The Maple command line to compute this solution directly using hyper3 is: $>$ dsolve(ode, [hyper3]); .

\section{An example of the ${ }_{1} F_{1}$ class}

As an example which also requires an extension of the algorithm to handle symbolic powers in the invariant (12), consider Kamke's second order linear equation 2.15:

$$
y^{\prime \prime}+\left(\mu x^{2 \sigma}+\nu x^{\sigma-1}\right) y=0
$$

where $\mu, \nu$ and $\sigma$ are constants with respect to $x$. This equation is already in normal form and the shifted invariant (25) for it is

$$
J_{1}(x)=1 / 4-x^{2}\left(\mu x^{2 \sigma}+\nu x^{\sigma-1}\right)
$$

To compute the values of $k$ entering (26) and leading to $J_{0}(x)$ with minimized integer powers, in (27), instead of restricting $\tilde{k}$ to be a rational number, we allow it to depend on symbolic variables. So we compute $\tilde{k}$ such that the set of exponents entering (35), $A:=\{2 \sigma+2, \sigma+1\}$, becomes a set of integers after multiplying each element of it by $\tilde{k}$, resulting in $^{9} \tilde{k}=1 /(\sigma+1)$. In summary, using $\left\{t=x^{\sigma+1}, u(t)=x^{\sigma / 2} y(x)\right\}$, Kamke's equation (34) can be obtained from the following equation, which is already in normal form and has an invariant with minimized integer degrees, free of symbolic powers

$$
u^{\prime \prime}=-\frac{\left(4 \mu t^{2}+4 \nu t+\sigma^{2}+2 \sigma\right)}{4(\sigma+1)^{2} t^{2}} u
$$

Proceeding with step 3 , the invariant is the coefficient of $u$ in the above and the degrees with respect to $t$ of its numerator and factors in its denominator match the Table 1 of sec.1.2 identifying (36) as equivalent to the ${ }_{1} \mathrm{~F}_{1}$ equation under Möbius transformations (6).

As in the previous example, in step 4, comparing the invariant of (36) with the invariant (18) of the transformed ${ }_{1} \mathrm{~F}_{1}$ equation, we compute the values of the parameters entering the ${ }_{1} \mathrm{~F}_{1}$ equation (3) such that the equivalence exists, as well as the parameters entering the Möbius transformation. Composing all the transformations, we arrive at the solution for Kamke's example 2.15

$$
\begin{aligned}
y= & \mathrm{e}^{\left(-\frac{i \sqrt{\mu} x^{\sigma+1}}{\sigma+1}\right)}( \\
& { }_{1} \mathrm{~F}_{1}\left(\frac{\sqrt{\mu} \sigma+i \nu}{2 \sqrt{\mu}(\sigma+1)} ; \frac{\sigma}{\sigma+1} ; \frac{2 i \sqrt{\mu}}{\sigma+1} x^{\sigma+1}\right) C_{1} \\
& \left.+{ }_{1} \mathrm{~F}_{1}\left(\frac{\sqrt{\mu}(\sigma+2)+i \nu}{2 \sqrt{\mu}(\sigma+1)} ; \frac{\sigma+2}{\sigma+1} ; \frac{2 i \sqrt{\mu}}{\sigma+1} x^{\sigma+1}\right) x C_{2}\right)
\end{aligned}
$$

where $C_{1}$ and $C_{2}$ are arbitrary constants. The time consumed by the implementation in Maple to perform these steps and return the solution above is again 0.4 seconds, as in the previous example. This also illustrates that, for typical problems, the additional handling of symbolic powers does not imply on any important performance cost.

\footnotetext{
${ }^{9}$ To perform this computation, it suffices to sequentially take the gcd between each of the elements of A.
} 


\section{ON THE COMPUTATION OF THE SEC- OND INDEPENDENT SOLUTION}

The algorithm presented is based on computing a transformation mapping a ${ }_{p} \mathrm{~F}_{q}$ equation into a given linear ODE, then applying that transformation to the solution of the ${ }_{p} \mathrm{~F}_{q}$ equation to obtain the solution for the given problem. This process has a subtlety: depending on the values of the hypergeometric parameters, we may have only one independent solution available for the ${ }_{p} \mathrm{~F}_{q}$ equation. In these cases, the second independent solution can be obtained through integration: if $y=S(x)$ is a solution of (1), then

$$
y=\int \frac{\mathrm{e}^{\left(\int A(x) d x\right)}}{S(x)^{2}} d x S(x)
$$

is a second independent solution directly computable from $S(x)$ and $A(x)$.

This approach, however, frequently introduces uncomputable integrals, thus complicating further manipulations and undermining the usefulness of the result. As an example of this situation, for the ${ }_{2} \mathrm{~F}_{1}$ equation,

$$
\left(x^{2}-x\right) y^{\prime \prime}+((a+b+1) x-c) y^{\prime}+b a y=0,
$$

the two independent solutions are:

$$
\begin{aligned}
y= & { }_{2} \mathrm{~F}_{1}(a, b ; c ; x) C_{1} \\
& +x^{1-c}{ }_{2} \mathrm{~F}_{1}(b-c+1, a-c+1 ; 2-c ; x) C_{2}
\end{aligned}
$$

but for $c=1$ these two solutions are equal. Using the integration recipe (38), a second independent solution is

$$
y=\int \frac{\mathrm{e}^{\left(\int \frac{(a+b+1) x-1}{x^{2}-x} d x\right)}}{{ }_{2} \mathrm{~F}_{1}(a, b ; 1 ; x)^{2}} d x{ }_{2} \mathrm{~F}_{1}(a, b ; 1 ; x)
$$

Although the inner integral, with rational integrand, is easy to compute, the outer integral, with ${ }_{2} \mathrm{~F}_{1}(a, b ; 1 ; x)^{2}$ in its denominator, is uncomputable in current computer algebra systems.

The approach used in hyper3 to minimize the occurrence of uncomputable integrals consists of exploring the group of automorphisms of the ${ }_{2} \mathrm{~F}_{1}$ equation in order to make $c$ not an integer when that is possible. Recalling, the group elements and their action are

\begin{tabular}{|l|c|}
\hline Group element & Action on the plane \\
\hline$g_{1}: x \rightarrow x$ & $(0 \rightarrow 0,1 \rightarrow 1, \infty \rightarrow \infty)$ \\
\hline$g_{2}: x \rightarrow 1-x$ & $(0 \rightarrow 1,1 \rightarrow 0, \infty \rightarrow \infty)$ \\
\hline$g_{3}: x \rightarrow 1 / x$ & $(0 \rightarrow \infty, 1 \rightarrow 1, \infty \rightarrow 0)$ \\
\hline$g_{4}: x \rightarrow 1 /(1-x)$ & $(0 \rightarrow 1,1 \rightarrow \infty, \infty \rightarrow 0)$ \\
\hline$g_{5}: x \rightarrow(x-1) / x$ & $(0 \rightarrow \infty, 1 \rightarrow 0, \infty \rightarrow 1)$ \\
\hline$g_{6}: x \rightarrow x /(x-1)$ & $(0 \rightarrow 0,1 \rightarrow \infty, \infty \rightarrow 1)$ \\
\hline
\end{tabular}

Table 2. Group of automorphisms of the ${ }_{2} F_{1}$ equation

These transformations, known to act as permutations on the set $\{0,1, \infty\}$, also act as permutations on a set $\{\lambda, \mu, \kappa\}$ related to the hypergeometric parameters $\{a, b, c\}$ by

$$
\lambda=1-c, \quad \mu=a+b-c, \quad \kappa=a-b
$$

These three parameters are the exponent differences of the normal form of the ${ }_{2} \mathrm{~F}_{1}$ equation (3), at $\{0,1, \infty\}$ respectively. The action of each $g_{i}$ on these parameters is obtained from Table 2 by respectively changing $\{0,1, \infty\}$ by $\{\lambda, \mu, \kappa\}$. Hence, the solution (40) can be written in different manners, by changing the application point of the ${ }_{2} \mathrm{~F}_{1}$ function using the $g_{i}$, permuting accordingly the parameters $\{\lambda, \mu, \kappa\}$ entering the ${ }_{2} \mathrm{~F}_{1}$ function and multiplying the result by the proper non-constant factor ${ }^{10}$.

For example, when $c$ is an integer but $a+b$ is not an integer, applying $g_{2}$ and permuting the parameters $\mu \leftrightarrow \lambda$, the power $x^{1-c}$ entering (40) becomes a power with noninteger exponent. Using this mechanism, for (39) at $c=1$, instead of the solution with integrals (41) we obtain two independent solutions free of uncomputed integrals:

$$
\begin{aligned}
y= & { }_{2} \mathrm{~F}_{1}(a, b ; a+b ; 1-x) C_{1} \\
& +(x-1)^{1-b-a}{ }_{2} \mathrm{~F}_{1}(1-b, 1-a ; 2-b-a ; 1-x) C_{2}
\end{aligned}
$$

When $c$ and $a+b$ are both integers, $g_{2}$ does not resolve the problem, but if $a-b$ is not an integer then $g_{3}$ does, since it permutes the integer $\lambda=1-c$ with the non-integer $\kappa=a-b$. For example, for $a=2 / 3, b=1 / 3, c=1$, (39) becomes

$$
2 y / 9+(2 x-1) y^{\prime}+\left(x^{2}-x\right) y^{\prime \prime}=0
$$

Applying $g_{3}$ and permuting the parameters $\lambda$ and $\kappa$, we obtain the following two independent solutions free of integrals

$$
\begin{aligned}
y= & x^{-1 / 3}{ }_{2} \mathrm{~F}_{1}(1 / 3,1 / 3 ; 2 / 3 ; 1 / x) C_{1} \\
& +x^{2 / 3}{ }_{2} \mathrm{~F}_{1}(2 / 3,2 / 3 ; 4 / 3 ; 1 / x) C_{2}
\end{aligned}
$$

When all of $c, a+b$ and $a-b$ are integers, these permutations are in principle of no use, but still for some cases the solution can be represented free of integrals. This is the case of Legendre's equation. Recalling the relationship between the associated Legendre function of the first kind and the hypergeometric ${ }_{2} \mathrm{~F}_{1}$ function ${ }^{11}$,

$$
\begin{aligned}
& \text { LegendreP }(a, b, z)= \\
& \qquad \frac{(z+1)^{1 / 2 b}{ }_{2} \mathrm{~F}_{1}(a+1,-a ; 1-b ;(1-z) / 2)}{(z-1)^{1 / 2 b} \Gamma(1-b)},
\end{aligned}
$$

whenever the group elements of Table 2 can map the ${ }_{2} \mathrm{~F}_{1}$ function solution into one of the form above, one independent solution can be expressed using LegendreP and the second one is obtained from the first one replacing LegendreP by the associated function of the second kind LegendreQ.

For example, for

$$
y / 4+(2 x-1) y^{\prime}+\left(x^{2}-x\right) y^{\prime \prime}=0
$$

we have $\mu=\kappa=\lambda=0$, so $c=1$ and both $a+b$ and $a-b$ are integers. A solution free of integrals is

$$
\begin{aligned}
y= & \text { LegendreP }(-1 / 2,2 x-1) C_{1} \\
& + \text { LegendreQ }(-1 / 2,2 x-1) C_{2}
\end{aligned}
$$

${ }^{10}$ These multiplicative factors are different for each $g_{1}$; we omit them here for brevity.

${ }^{11}$ We use here the Maple convention for the branch cuts of LegendreP; the idea being discussed is independent of that. 


\section{Conclusions}

In this presentation we discussed an algorithm for second order linear ODEs, we called it hyper3, for computing nonLiouvillian solutions by resolving an equivalence to the ${ }_{2} \mathrm{~F}_{1}$, ${ }_{1} \mathrm{~F}_{1}$ and ${ }_{0} \mathrm{~F}_{1}$ equations. Taking Kamke's book as testing arena, this algorithm is the most successful one of the current set of linear ODE algorithms of the Maple system. From the 363 corresponding examples of Kamke's book having rational coefficients, hyper3 alone solves 331 (91\%), followed by Kovacic's algorithm solving 181 (50 \%). Moreover, from these 181 examples admitting Liouvillian solutions, hyper3 solves $163(90 \%)$.

The fact that, for $90 \%$ of these equations admitting Liouvillian solutions, the solution can also be computed as a hypergeometric one of the form (9) is a good indication that the restriction used to make the algorithm feasible is appropriate. The fact that around one half of Kamke's examples only admit special function solutions of non-Liouvillian form also illustrates the relevance of this type of solution in the general framework of linear ODE problems popping up in applications.

Despite the simplicity of the approach, till the end of 2001, when the routines for this algorithm were developed, no equivalent or similar algorithms were available in any of the Axiom, Maple, Mathematica, MuPAD or Reduce computer algebra systems (CAS). These CAS failed in computing special function solutions but for occasional success, e.g., by previous to hyper3 Maple routines able to resolve an equivalence under only power transformations of the form (7) [13, or an equivalence under only Möbius transformations and only with respect to the ${ }_{2} \mathrm{~F}_{1}$ class [14.

Since at the core of hyper3 there is the concept of singularities, two natural extensions of this work consist of applying the same ideas to compute solutions for linear ODEs of order three and higher [15] and for second order equations of Heun type. The latter have four regular singular points or any combination of singularities derived from that case through confluence processes [16; one example of these are Mathieu equations. Related work is in progress [17, 18.

\section{Acknowledgments}

This work was supported by the MITACS NCE project, the Centre of Experimental and Constructive Mathematics of Simon Fraser University, and the NSERC of Canada. We would like to thank one of the referees for her/his kind, motivating and illustrated comments.

\section{REFERENCES}

[1] Seaborn J.B., "Hypergeometric Functions and Their Applications", Text in Applied Mathematics, 8, Springer-Verlag (1991).

[2] M. Abramowitz and I. A. Stegun, "Handbook of mathematical functions", Dover (1964).

[3] L. Chan supervised by E.S. Cheb-Terrab. "On Solving second order linear ODEs admitting non-Liouvillian solutions." Report for NSERC University research award, Department of Mathematics, Simon Fraser University (2001).

[4] N. Kamran and P.J. Olver. "Equivalence of Differential Operators." SIAM J. Math. Anal. 20, no. 5, 1172 (1989).
[5] M. Petkovsek and B. Salvy. "Finding All Hypergeometric Solutions of Linear Differential Equations." Proceedings of ISSAC '93, Edited by M. Bronstein. ACM Press, 27-33 (1993).

[6] K. von Bülow, "Equivalence methods for second order linear differential equations", M.Sc. Thesis, Faculty of Mathematics, University of Waterloo (2000).

[7] B. Willis, "An extensible differential equation solver for computer algebra", SIGSAM, March (2001).

[8] M. Bronstein and S. Lafaille, "Solutions of linear ordinary differential equations in terms of special functions", Proceedings of ISSAC'02, Lille, ACM Press, 23-28 (2002).

[9] E.L. Ince, "Ordinary Differential Equations", Dover Publications (1956).

[10] P. Olver, "Equivalence, Invariants and Symmetry", Cambridge Unversity Press (1995).

[11] E.W. Weisstein, "Concise Encyclopedia of Mathematics", second edition, CRC Press (1999).

[12] E. Kamke, "Differentialgleichungen", N.Y. Chelsea Publ. Co. (1947).

[13] G. Labahn. "Methods for Solving Linear ODEs in Maple." University of Waterloo Tech Report (2000).

[14] M. van Hoeij, http://web.math.fsu.edu/ hoeij/daisy/lib/DEtools/src/RiemannPsols (1997).

[15] E.S. Cheb-Terrab, "ODE trends in computer algebra: four linear and nonlinear challenges", proceedings of the Maple Summer Workshop, Waterloo, Canada (2002).

[16] S.Y. Slavyanov and W.Lay. "Special Functions, A Unified Theory Based on Singularities", Oxford Mathematical Monographs (2000).

[17] E.S. Cheb-Terrab, "Computing Mathieu function solutions for linear ODEs", http://lie.uwaterloo.ca/odetools/mathieu_function_solutions.html (2003).

[18] E.S. Cheb-Terrab, D. Frenkel, R.Portugal, "Mathieu equations: series and exact solutions", in preparation (2004). 